psychologists and paediatricians was extremely limited; she continued to lose weight whilst in hospital.

However, the patient's family mentioned some history which suggested a possible inflammatory bowel disease. Stool calprotectin was $>1800 \mathrm{mcg} / \mathrm{g}$.

Following an MDT meeting, psychiatry started aripiprazole to help her anxiety symptoms. Input from Youth Work/Play Therapy helped the patient to undergo blood tests, the results of which supported a diagnosis of inflammatory bowel disease. A referral was made to Paediatric Gastroenterology, who organised an endoscopy; the endoscopy showed focal active colitis with mucosal ulceration consistent with Crohn's disease, confirmed on histology. Upper GI endoscopy showed chronic gastritis, positive for Helicobacter pylori.

Treatment for Crohn's disease was commenced with methylprednisolone and infliximab. She also received Helicobacter eradication therapy. The patient continued on aripiprazole, and was able to engage with CAMHS whilst an inpatient. She was discharged after 5 weeks in hospital. She continues to receive treatment from both CAMHS and Gastroenterology, and is doing well.

Conclusion Whilst diagnostic parsimony remains a prudent approach to diagnosis, patients may have multiple pathologies contributing to the same presentation. Clinicians should keep an open mind regarding the possibility of co-existing illnesses, even once the first diagnosis has been confirmed.

\section{G529(P) AN EXPLORATORY ANALYSIS OF BARRIERS TO ACCESSING HEALTH CARE AMONG YOUNG PEOPLE IN 30 LOW-MIDDLE INCOME COUNTRIES}

N Nachiappan, D Hargreaves. School of Public Health, Imperial College London, London, UK

10.1136/archdischild-2020-rcpch.447

Aims The importance of good health in young people and the currently poor health outcomes of this group are well documented. Qualitative studies in Low and Middle Income Countries (LMICs) indicate that these poor outcomes are partly due to young people facing additional healthcare barriers compared to other age groups and that tackling these barriers may make an important contribution in improving young people's health. This paper aimed to investigate the prevalence of healthcare barriers in a quantitative manner amongst young people (10-24) and older adults (25-49) across 30 LMICs to identify if young people face additional healthcare barriers compared to older adults.

Methods Secondary analyses of survey data from young people (10-24) and older adults (25-49) across 30 countries was performed using 6 selected survey questions within the Demographic and Heath Surveys, a multinational database.

Results On average, young people reported significantly greater barriers to accessing evidence-based care compared to older adults across all survey questions measured with an average of 8.4 percentage point difference across the 6 questions. During question specific analysis, wide variation was seen between countries, with some countries reporting insignificance between the two groups. The range seen between countries varied between questions, with a difference of up to 33 percentage points between younger people and older adults. 7 countries showed no differences between groups across 2 or more survey questions. In particular, Rwanda showed insignificance across all survey questions data was present for. A more positive response to a question by a country does not appear to correlate with insignificance between younger and older adults.

Conclusion Our results, whilst supporting the previously published qualitative data that young people in LMICs face additional health barriers, clearly highlights a wide variation between countries. This indicates country specific barriers to accessing healthcare, which prevent young people having optimum use of the resources available. The insignificance between groups in certain countries indicate that barriers to healthcare access for young people are not inevitable. This report highlights the need for more in-depth country specific analyses of healthcare barriers and further research into causative factors that can affect healthcare access for young people.

\section{G530(P) DO YOUNG PEOPLE WITH EPILEPSY DISENGAGE WITH HEALTHCARE FOLLOWING TRANSITION TO ADULT SERVICES?}

J McInally, M Kirkpatrick. Paediatrics, NHS Tayside, Dundee, UK

\subsection{6/archdischild-2020-rcpch.448}

Introduction There is a concern that young people wit epilepsy will disengage with healthcare following transition to adult services. The aim of this review was to identify from the medical literature any evidence to support or refute these concerns.

Methods Markers of engagement were identified as scheduled care attendance, unscheduled care attendance, medication adherence, mortality, healthcare costs and health behaviours. A literature review was carried out for each of these areas. Studies published in the period 2008-2018 were included.

Results There is good evidence that there is no increase in emergency care use in young adults with epilepsy compared to children or older adults. There are no studies comparing scheduled care use by children, young people and older adults. There is evidence that young people with epilepsy attend their GP twice as frequently as age matched peers, but it is unclear how much of this is related to epilepsy and how much to co-morbidity. There is conflicting evidence around medication adherence and therefore no conclusion can be drawn as to whether transition has an impact on this.

Young adults with epilepsy have higher rates of unexpected death than children or older adults. They are more likely to have uncontrolled epilepsy than older adults. In a deprived population, death by accident is more likely in adults with epilepsy. People with childhood epilepsy have been found to have a lower level of education than age matched controls, as well as higher unemployment levels and increased reliance on state welfare programmes.

Conclusion There is a paucity of evidence to show whether or to what extent young people with epilepsy disengage with healthcare following transition to adult services. There is no evidence that young people have different scheduled care attendance or medication adherence following transition. There is good evidence that young adults do not have higher emergency care attendance or admissions to hospital. There is evidence that young people have an increased risk of unexpected death around the time of transition and this should be addressed. 